00-000

\title{
Mass parameters for relative and neck collective motions in heavy ion fusion reactions
}

\author{
Kai Zhao, ${ }^{1}$ Zhuxia Li, ${ }^{1, *}$ Xizhen $\mathrm{Wu},{ }^{1, \dagger}$ and Zhixiang Zhao ${ }^{1}$ \\ ${ }^{1}$ China Institute of Atomic Energy, Beijing 102413, P. R. China
}

(Dated: March 2, 2022)

\begin{abstract}
Mass parameters for the relative and neck motions in fusion reactions of symmetric systems ${ }^{90} \mathrm{Zr}+{ }^{90} \mathrm{Zr},{ }^{110} \mathrm{Pd}+{ }^{110} \mathrm{Pd}$, and ${ }^{138} \mathrm{Ba}+{ }^{138} \mathrm{Ba}$ are studied by means of a microscopic transport model. The shape of the nuclear system is determined by an equi-density surface obtained from the density distribution of the system. The relative and neck motions are then studied and the mass parameters for these two motions are deduced. The mass parameter for the relative motion is around the reduced mass when the reaction partners are at the separated configuration and increases with decrease of the distance between two reaction partners after the touching configuration. The mass parameter for the neck motion first decreases slightly up to the touching configuration and then increases with the neck width, and its magnitude is from less than tenth to several times more than the total mass of the system. The mass parameters obtained from the microscopic transport model are larger than the ones obtained from the hydrodynamic model and smaller than those obtained from the linear response function theory. The mass parameters for both motions depend on the reaction systems, but the one for the relative motion depends on the incompressibility of the EoS more obviously than that for neck motion.

PACS numbers: 25.70.-z, 24.10.-i
\end{abstract}

*Electronic address: lizwux@.ciae.ac.cn

${ }^{\dagger}$ Electronic address: lizwux9@ciae.ac.cn 


\section{INTRODUCTION}

In the study of a large amplitude collective motion in nuclear systems including heavy ion fusion reactions( especially the synthesis of superheavy nuclei) and fission, the macroscopic model plays a very important role, in which a few collective degrees of freedom are usually used to describe the complex dynamic process. The potential energy surface, the mass parameter and the viscosity are the most important quantities in the macroscopically description of the large amplitude collective motion. Many works have been done for the study of these three quantities. The importance of the potential energy surface in the nuclear large amplitude collective motion such as in fission and heavy ion fusion process is well known and up to now the calculation of potential energy surface based on the macroscopic and microscopic method seems to be quite successful $[1,2,3,4]$. But the problem of kinetic energy ,i.e. the mass parameter, is far less settled than that of potential energies. As a matter of fact, the dynamical behavior of nuclear systems with large-amplitude collective motions, such as fusion and fission not only depends on the potential energy surface but also on mass parameter. Take a most simple example, let us consider the fusion process as a barrier penetration problem. In the WKB formula for barrier penetration the inertia tensor $\mathrm{M}(\mathrm{R})$ appears in the action integral $\mathrm{S}(\mathrm{L})=\mp \frac{2}{\hbar} \int_{L}(2 M(R)(U(R)-E))^{1 / 2} d R$ along the fusion path L. The $\mathrm{U}(\mathrm{R})$ and $\mathrm{E}$ are the potential energy surface and the center of mass energy, respectively. The sign '-' and '+' refer to above and below the barrier energies. For simplicity only the internuclear distance $\mathrm{R}$ is taken to be the collective coordinate. The probability for penetrating the barrier(may be a multi-dimension barrier) is calculated as usual by $\mathrm{P}(\mathrm{L})=\exp (-\mathrm{S}(\mathrm{L}))$. In many studies the $\mathrm{M}(\mathrm{R})$ was simply taken to be the reduced mass. This simple approximation is only partly valid for fusion reaction of light nuclei. For fusion reactions of heavy nuclei, it is well known that not only the relative collective motion but also the neck motion becomes very important. The process for penetrating the barrier becomes more complicated and the $\mathrm{M}(\mathrm{R})$ can not be simply taken to be the reduced mass. Thus, the impact of the mass parameter on fusion process for heavy systems becomes incontestable. Recently renewed interest in the study of mass parameter is motivated by the study of the synthesis of superheavy nuclei $[5,6,7,8]$. The qualitative and quantitative study of the mass parameter becomes more important. In the dynamic study of synthesis of superheavy nuclei the mass parameters for collective motions were studied by the cranking 
model( or by the linear response function theory(LRFT) based on the cranking model) and the hydrodynamical model. In [5, 6], a large mass parameter for neck motion was found by means of the LRFT method leading to a restriction for a growing neck in dinuclear system and melting of the dinuclear system along the internuclear distance into a compound system. While in [7] a hydrodynamical mass parameter was adopted, then a subsequent shape evolution of pear shaped mono-nucleus formed with two incident ions could happen. Thus, the rather different mass parameters obtained by cranking and hydrodynamical models lead to complete different description of the fusion mechanism for heavy systems due to different fusion paths. The cranking model was developed to calculate the mass parameter of nuclear systems microscopically $[9,10,11,12,13,14]$. But the abrupt change of mass parameters near the level crossing makes difficulties for practical usage[15]. Whereas for the hydrodynamical model, the assumption of the irrotational flow of an incompressible and non-viscous fluid $[7,16,17,18,19]$ is too simple for many practical purposes. Furthermore, in both approaches, one usually adopts a way that only one collective degree of freedom is taken to be a variable and the others are fixed in the calculation of mass parameters. The condition of the validity of this (static) treatment needs to be tested because in a real heavy ion fusion reaction all degrees of freedom of collective motion change dynamically and self-consistently. Therefore, it seems to be necessary to investigate the mass parameters for the collective motion in fusion reactions by a more realistic dynamic model. In this work for the first time we try to employ the microscopic transport model, namely the Improved Quantum Molecular Dynamics (ImQMD) model to investigate the mass parameters for collective motions in heavy ion fusion reactions and possibly to find out the conditions for the validity of the widely used static treatment in the calculations of mass parameter.

The quantum molecular dynamics (QMD) model being successfully used in intermediate energy heavy-ion collisions was extended to apply to heavy ion collisions at energies near barrier by making a series of improvements[20, 21]. The main improvements introduced are: introducing the surface and surface symmetry energy terms in the potential energy density functional, introducing the phase space occupation constraint[22] which can be considered as an approximate treatment of anti-symmetrization, and a system size dependent wave packet width. Furthermore, considering the especially importance of a proper initial condition for the application of the microscopic transport model to low energy heavy ion reactions, in the ImQMD model the requirement of the projectile and target at initial time being at 
ground states is enforced. And consequently the projectile and target can be stable for a long enough time and the structure effect in entrance can be taken into account partly. Thus, in principle, the dissipation, diffusion and correlation effects are all included without introducing any freely adjusting parameter. With the ImQMD model the fusion dynamics at energies near and above the barrier has extensively been studied. Firstly, the stable initial nuclei with good ground-state properties such as the binding energies, root-mean-square radii and density distribution for a series of nuclei from ${ }^{16} \mathrm{O}$ to ${ }^{208} \mathrm{~Pb}$ and ${ }^{238} \mathrm{U}$ were realized within several thousands fm/c $[20,23]$. The Coulomb barriers for many reaction partners can be described well. The model were applied to calculate fusion (capture) excitation functions for a series of fusion reactions including neutron-rich projectile and target [21]. We also extend the ImQMD model to study the capture cross sections for the reactions ${ }^{48} \mathrm{Ca}+{ }^{208} \mathrm{~Pb},{ }^{238} \mathrm{U}$ leading to super-heavy nuclei [24]. The calculated results are in good agreement with experimental data. Thus, it is more confident to apply the ImQMD model to study the mass parameters for collective motions in heavy ion fusion reactions. In this work, the mass parameters for symmetric reactions of ${ }^{90} \mathrm{Zr}+{ }^{90} \mathrm{Zr},{ }^{110} \mathrm{Pd}+{ }^{110} \mathrm{Pd}$, and ${ }^{138} \mathrm{Ba}+{ }^{138} \mathrm{Ba}$ are investigated. The paper includes following parts: In section II we briefly introduce the ImQMD model. In section III, we present the results of our study on the mass parameters for ${ }^{90} \mathrm{Zr}+{ }^{90} \mathrm{Zr},{ }^{110} \mathrm{Pd}+{ }^{110} \mathrm{Pd}$, and ${ }^{138} \mathrm{Ba}+{ }^{138} \mathrm{Ba}$. Finally, we give brief summary and discussion in section IV.

\section{BRIEF INTRODUCTION OF THE IMQMD MODEL}

In the ImQMD model as the same as the original QMD model, each nucleon is represented by a coherent state of a Gaussian wave packet [25, 26],

$$
\phi_{i}(\mathbf{r})=\frac{1}{\left(2 \pi \sigma_{r}^{2}\right)^{3 / 4}} \exp \left[-\frac{\left(\mathbf{r}-\mathbf{r}_{i}\right)^{2}}{4 \sigma_{r}^{2}}+\frac{i}{\hbar} \mathbf{r} \cdot \mathbf{p}_{i}\right],
$$

where, $\mathbf{r}_{i}, \mathbf{p}_{i}$, are the center of $\mathrm{i}$-th wave packet in the coordinate and momentum space, respectively. $\sigma_{r}$ represents the spatial spread of the wave packet. Through a Wigner transformation, the one-body phase space distribution function for $\mathrm{N}$-distinguishable particles is given by:

$$
f(\mathbf{r}, \mathbf{p})=\sum_{i} \frac{1}{(\pi \hbar)^{3}} \exp \left[-\frac{\left(\mathbf{r}-\mathbf{r}_{i}\right)^{2}}{2 \sigma_{r}^{2}}-\frac{2 \sigma_{r}^{2}}{\hbar^{2}}\left(\mathbf{p}-\mathbf{p}_{i}\right)^{2}\right]
$$


The density and momentum distribution functions of a system read

$$
\begin{aligned}
& \rho(\mathbf{r})=\int f(\mathbf{r}, \mathbf{p}) d \mathbf{p}=\sum_{i} \rho_{i}(\mathbf{r}), \\
& g(\mathbf{p})=\int f(\mathbf{r}, \mathbf{p}) d \mathbf{r}=\sum_{i} g_{i}(\mathbf{p}),
\end{aligned}
$$

respectively, where the sum runs over all particles in the system. $\rho_{i}(\mathbf{r})$ and $g_{i}(\mathbf{p})$ are the density and momentum distributions of nucleon i:

$$
\begin{aligned}
\rho_{i}(\mathbf{r}) & =\frac{1}{\left(2 \pi \sigma_{r}^{2}\right)^{3 / 2}} \exp \left[-\frac{\left(\mathbf{r}-\mathbf{r}_{i}\right)^{2}}{2 \sigma_{r}^{2}}\right], \\
g_{i}(\mathbf{p}) & =\frac{1}{\left(2 \pi \sigma_{p}^{2}\right)^{3 / 2}} \exp \left[-\frac{\left(\mathbf{p}-\mathbf{p}_{i}\right)^{2}}{2 \sigma_{p}^{2}}\right],
\end{aligned}
$$

where $\sigma_{r}$ and $\sigma_{p}$ are the widths of wave packets in coordinate and momentum space, respectively, and they satisfy the minimum uncertainty relation:

$$
\sigma_{r} \sigma_{p}=\frac{\hbar}{2}
$$

The propagation of nucleons under the self-consistently generated mean field is governed by Hamiltonian equations of motion:

$$
\dot{\mathbf{r}}_{i}=\frac{\partial H}{\partial \mathbf{p}_{i}}, \dot{\mathbf{p}}_{i}=-\frac{\partial H}{\partial \mathbf{r}_{i}}
$$

Hamiltonian $H$ consists of the kinetic energy and effective interaction potential energy,

$$
H=T+U_{l o c}+U_{\text {Coul }} .
$$

Here, $U_{\text {Coul }}$ is the Coulomb energy, and $U_{l o c}=\int V_{l o c}[\rho(\boldsymbol{r})] d \boldsymbol{r} . \quad V_{l o c}[\rho(\boldsymbol{r})]$ is the nuclear potential energy density functional, which is introduced according to Skyrme interaction energy density functional with spin-orbit term omitted [21, 27]. It reads

$$
V_{l o c}[\rho]=\frac{\alpha}{2} \frac{\rho^{2}}{\rho_{0}}+\frac{\beta}{\gamma+1} \frac{\rho^{\gamma+1}}{\rho_{0}^{\gamma}}+\frac{g_{\text {sur }}}{2 \rho_{0}}(\nabla \rho)^{2}+\frac{C_{s}}{2 \rho_{0}}\left(\rho^{2}-\kappa_{s}(\nabla \rho)^{2}\right) \delta^{2}+g_{\tau} \frac{\rho^{\eta+1}}{\rho_{0}^{\eta}} .
$$

Here, $\delta=\left(\rho_{n}-\rho_{p}\right) /\left(\rho_{n}+\rho_{p}\right)$ is the isospin asymmetry and the $\rho, \rho_{n}, \rho_{p}$ are the nucleon, neutron, and proton density, respectively. By integrating $V_{l o c}$, we obtain the local interaction potential energy: 


$$
\begin{aligned}
U_{l o c}= & \frac{\alpha}{2} \sum_{i} \sum_{j \neq i} \frac{\rho_{i j}}{\rho_{0}}+\frac{\beta}{\gamma+1} \sum_{i}\left(\sum_{j \neq i} \frac{\rho_{i j}}{\rho_{0}}\right)^{\gamma} \\
& +\frac{g_{\text {sur }}}{2} \sum_{i} \sum_{j \neq i} f_{s i j} \frac{\rho_{i j}}{\rho_{0}}+g_{\tau} \sum_{i}\left(\sum_{j \neq i} \frac{\rho_{i j}}{\rho_{0}}\right)^{\eta}+\frac{C_{s}}{2} \sum_{i} \sum_{j \neq i} t_{i} t_{j} \frac{\rho_{i j}}{\rho_{0}}\left(1-\kappa_{s} f_{s i j}\right),
\end{aligned}
$$

where

$$
\begin{gathered}
\rho_{i j}=\frac{1}{\left(4 \pi \sigma_{r}^{2}\right)^{3 / 2}} \exp \left[-\frac{\left(\mathbf{r}_{i}-\mathbf{r}_{j}\right)^{2}}{4 \sigma_{r}^{2}}\right], \\
f_{s i j}=\frac{3}{2 \sigma_{r}^{2}}-\left(\frac{\mathbf{r}_{i}-\mathbf{r}_{j}}{2 \sigma_{r}^{2}}\right)^{2},
\end{gathered}
$$

and $t_{i}=1$ and -1 for proton and neutron, respectively.

The Coulomb energy can be written as the sum of the direct and the exchange contribution

$$
U_{\text {Coul }}=\frac{1}{2} \iint \rho_{p}(\mathbf{r}) \frac{e^{2}}{\left|\mathbf{r}-\mathbf{r}^{\prime}\right|} \rho_{p}\left(\mathbf{r}^{\prime}\right) d \mathbf{r} d \mathbf{r}^{\prime}-e^{2} \frac{3}{4}\left(\frac{3}{\pi}\right)^{1 / 3} \int \rho_{p}^{4 / 3} d \mathbf{r} .
$$

The collision term and the constraint for single particle occupation number are considered as the same as that in $[20,21,22]$.

To have a proper initial condition ( with good properties of projectile and target nuclei) is of crucial importance for studying low energy heavy ion reactions by means of the transport model description. In this work we pay special attention to the initial condition. The procedure for making initial nuclei is similar as that in ref. [20]. The initial nuclei applied in the study of reaction process have good ground state properties such as binding energies and rms radii and furthermore, their time evolution is very stable remaining almost unchanged for a long enough time(in this work, it is taken to be $2000 \mathrm{fm} / \mathrm{c}$ ). At the same time they are required to be without spurious particle emission. For the self-consistency, we adopt the same effective nuclear potential energy density in making initial nuclei and the simulation of the reaction process when solving the Hamiltonian equation(8). Two sets of interaction force parameters used in the calculations and the corresponding properties of saturated nuclear matter are given in table I. In this work, the definition of a fusion event is taken to be the same as that in [20], in which a fusion event is defined operationally like in TDHF calculations that is that the fusion event is defined rather operationally as the event in which 
the coalesced one-body density survives through one or more rotations of composite system or through several oscillations of its radius.

\begin{tabular}{ccccccccccc}
\hline \multicolumn{2}{c}{$\alpha(\mathrm{MeV})$} & $\beta(\mathrm{MeV})$ & $\gamma$ & $g_{\text {sur }}\left(\mathrm{MeVfm} m^{2}\right)$ & $g_{\tau}(\mathrm{MeV})$ & $\eta$ & $c_{s}(\mathrm{MeV})$ & $\kappa_{s}\left(\mathrm{fm}^{2}\right)$ & $\rho_{0}\left(\mathrm{fm}^{-3}\right)$ & $K_{\infty}(\mathrm{MeV})$ \\
\hline IQ1 & -310 & 258 & $7 / 6$ & 19.8 & 9.5 & $2 / 3$ & 32 & 0.08 & 0.165 & 144 \\
\hline IQ2 & -356 & 303 & $7 / 6$ & 7.0 & 12.5 & $2 / 3$ & 32 & 0.08 & 0.165 & 175 \\
\hline
\end{tabular}

TABLE I: the model parameters

\section{THE MASS PARAMETERS FOR COLLECTIVE MOTIONS IN HEAVY ION FUSION REACTIONS}

In the macroscopic model, the low energy heavy ion fusion reactions are generally described by five collective coordinates, namely the distance between the centers of mass of two colliding nuclei, the mass asymmetry degree of freedom, the neck parameter and deformation coordinates of two ends of the colliding system. Since in the early stage of reactions the mass parameters for relative collective motion and neck motion are weakly dependent on the deformations of two end parts[6], one often considers only three collective modes, i.e., the relative motion of two nuclei, the formation and rupture of neck and nucleon transfer between projectile and target. The mass parameters for those collective motion modes are expressed by a matrix and the corresponding collective kinetic energy is given by

$$
T=\frac{1}{2} \sum_{i j} M_{i j}(q) \dot{q}_{i} \dot{q}_{j} .
$$

Here, $M_{i j}$ is the mass parameter matrix and $q_{i}$ is the i-th collective coordinate. The nuclear mass parameters reflect the properties of a nuclear system related to the collective kinetic motion, which depend on the time dependent change of the shape of nuclear system and may depend on the effective nucleon-nucleon interaction. For the symmetric reactions considered in this work the mass asymmetric motion plays a negligible role and thus we can only consider the relative motion of projectile and target and the neck motion in the following study. 
A. The mass parameter for the relative motion of projectile(like) and target(like)

Let us first investigate the mass parameter for relative motion of projectile and target nuclei. In order to study the collective motion with the microscopic transport model one has to first define the collective coordinate by means of the microscopic quantities. The distance between the centers of mass of two nuclei $R$ can be expressed as

$$
R=A^{-1} \int z \cdot \operatorname{sign}(z) f(\vec{r}, \vec{p}) d \vec{r} d \vec{p}
$$

Here, $\operatorname{sign}(z)=\left\{\begin{array}{c}+1, \quad \mathrm{z}>0 ; \\ -1, \quad \mathrm{z}<0 .\end{array}\right.$ and the $f(\vec{r}, \vec{p})$ is the distribution function in phase space. The distance between the centers of mass of two nuclei $R$ and the neck width $\Delta$ (which will be discussed in the next subsection) for an axial symmetric nuclear system are sketched in Fig.1. In addition, the system length $\mathrm{L}$ is also shown in Fig.1. Within the ImQMD model approach, the conjugate momentum of the relative motion of two nuclei is expressed as

$$
P_{R}=\int P_{z} \cdot \operatorname{sign}(z) f(\vec{r}, \vec{p}) d \vec{r} d \vec{p}
$$

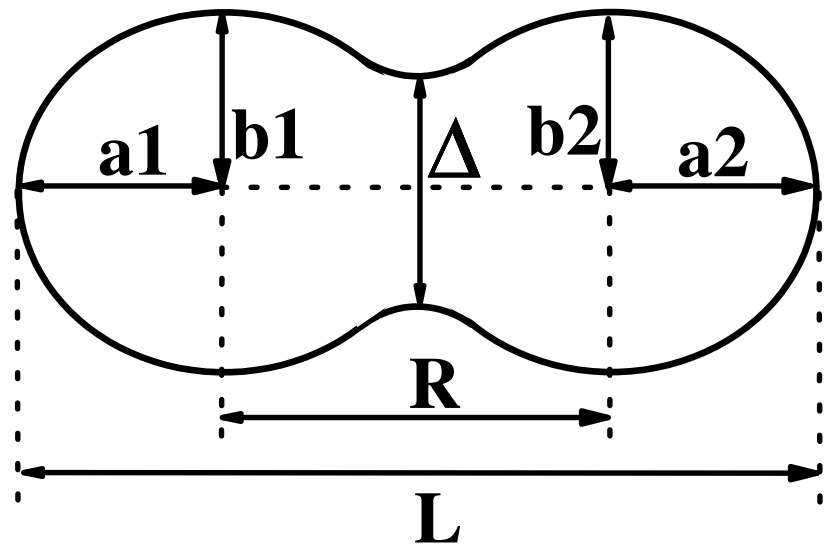

FIG. 1: (Color online) The schematic figure of deformation parameters of a reaction system.

The velocity of the relative motion of two nuclei

$$
\dot{R}=\frac{\Delta R}{\Delta t}
$$

can be obtained numerically. The mass parameter for relative collection motion of two nuclei is then calculated by

$$
M_{R R}=\frac{P_{R}}{\dot{R}}
$$


This formula seems to be questionable when the $\dot{R}$ approaches to zero, but, in fact, the $P_{R}$ also approaches to zero as the $\dot{R} \rightarrow 0$. This situation only happens at the fusion reaction closing to the end, which is a not interesting case.

In this work the fusion systems of ${ }^{90} \mathrm{Zr}+{ }^{90} \mathrm{Zr},{ }^{110} \mathrm{Pd}+{ }^{110} \mathrm{Pd},{ }^{138} \mathrm{Ba}+{ }^{138} \mathrm{Ba}$ are chosen for studying mass parameters. They are all along the $\beta$-stability line. The head on collisions at the incident energy of 1.1 times the height of Bass barrier[29] are considered for all systems. The incident energy is selected in such a way that the fusion process can be proceeded, whereas the compress or expand of nuclear matter does not appear.



FIG. 2: (Color online)The mass parameters for the relative collection motion as a function of $R / R_{0}$ for systems of ${ }^{90} \mathrm{Zr}+{ }^{90} \mathrm{Zr},{ }^{110} \mathrm{Pd}+{ }^{110} \mathrm{Pd}$ and ${ }^{138} \mathrm{Ba}+{ }^{138} \mathrm{Ba}$.

Fig.2 shows the dependence of the mass parameter $M_{R R}$ in the unit of total mass of the reaction system $M_{0}$ on the scaled distance between the centers of mass of two nuclei $R / R_{0}$ for systems of ${ }^{90} \mathrm{Zr}+{ }^{90} \mathrm{Zr},{ }^{110} \mathrm{Pd}+{ }^{110} \mathrm{Pd}$ and ${ }^{138} \mathrm{Ba}+{ }^{138} \mathrm{Ba}$ calculated with IQ2. Here $R_{0}=$ $r_{0}\left(A_{1}+A_{2}\right)^{1 / 3} \mathrm{fm}$ is the radius of the spherical compound nucleus formed by two nuclei. The $r_{0}$ is taken to be $1.16 \mathrm{fm}$. The corresponding configurations of the reaction system at different stages are also shown in the figure. The structures appeared in the $M_{R R}$ in the figure, especially for ${ }^{138} \mathrm{Ba}+{ }^{138} \mathrm{Ba}$ is mainly due to not enough number of the fusion events. From the figure one sees that when $R>1.8 R_{0}$ the mass parameter $M_{R R}$ approaches to 0.25 total mass of the system, i. e., the reduced mass, whereas in the case of $R<1.8 R_{0}$ the $M_{R R}$ increases with decrease of the distance $\mathrm{R}$. Another important feature is that the mass parameter for relative motion of two nuclei depends on the size of the reaction systems. The 
heavier system has the steeper increasing slope of $M_{R R}$ with decrease of $R$.

The incident energy dependence of mass parameters is also interesting. Here we only study the energy dependence of the $M_{R R}$. Fig.3 shows the mass parameter for relative motion for ${ }^{110} \mathrm{Pd}+{ }^{110} \mathrm{Pd}$ at $\mathrm{E}=1.0,1.1$ and $1.5 \mathrm{~V}_{B}$, where $\mathrm{V}_{B}$ refers to the height of the Bass barrier. The figure shows that the mass parameter for relative motion depends on the incident energy. For lower energy like $\mathrm{E}=1.0 \mathrm{~V}_{B}$, the mass parameter for relative motion changes slowly since the reaction process is slow and the reaction system has time to change their shapes gradually and consequently the mass parameter for relative motion increases gradually from reduced mass to larger than the reduced mass. As the incident energy increases the reaction process becomes faster and there is less time to change the shape of the system and the mass parameter for relative collective can be close to the reduced mass for a period of time and then increases rapidly at very late time. So we see from the figure that at higher energy such as at $\mathrm{E}=1.5 \mathrm{~V}_{B}$ the $M_{R R}$ is roughly equal to reduced mass when $\mathrm{R} / \mathrm{R}_{0} \geq 1.3$. But we expect it could be the case only for not heavy nuclear systems and at very above the barrier energies. The time evolution of the mass parameters for relative motion at three energies is shown in the inserted figure of Fig.3.

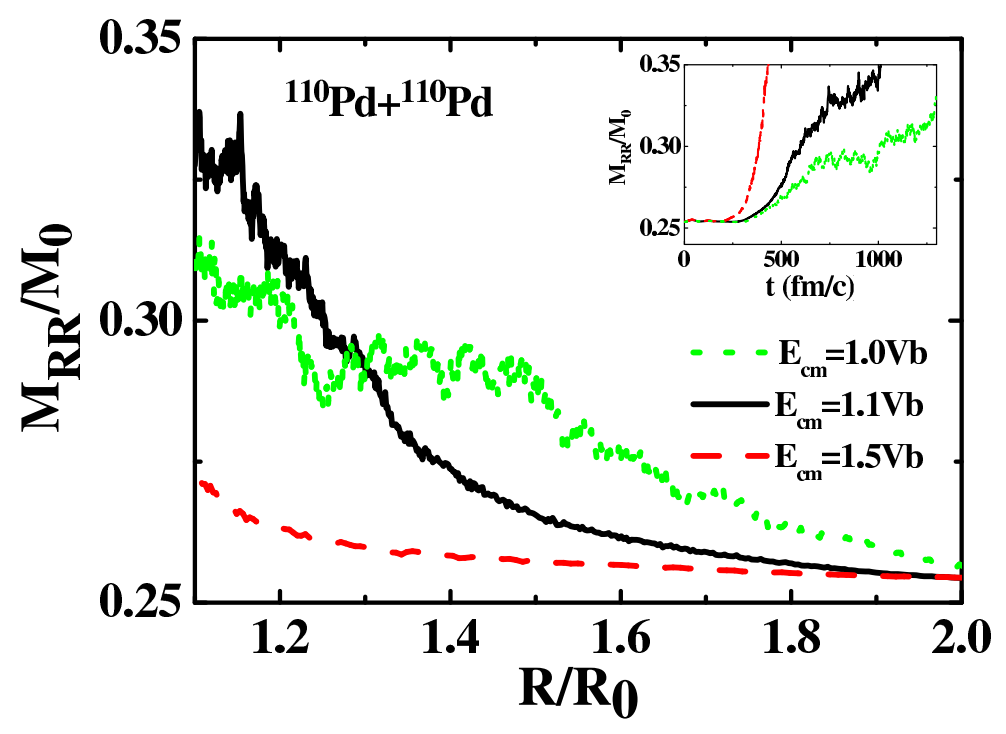

FIG. 3: (Color online)The incident energy dependence of the $M_{R R}$ for ${ }^{110} \mathrm{Pd}+{ }^{110} \mathrm{Pd}$ at $\mathrm{E}=1.0,1.1,1.5 \mathrm{~V}_{B}$. The inserted figure is the time evolution of the $M_{R R}$ for the same system.

Now let us investigate whether the mass parameter for relative motion depends on the nucleon-nucleon effective interaction. In Fig.4 we make a comparison between the mass parameters $M_{R R}$ calculated with two force parameter sets IQ1 and IQ2 (listed in Table I) 


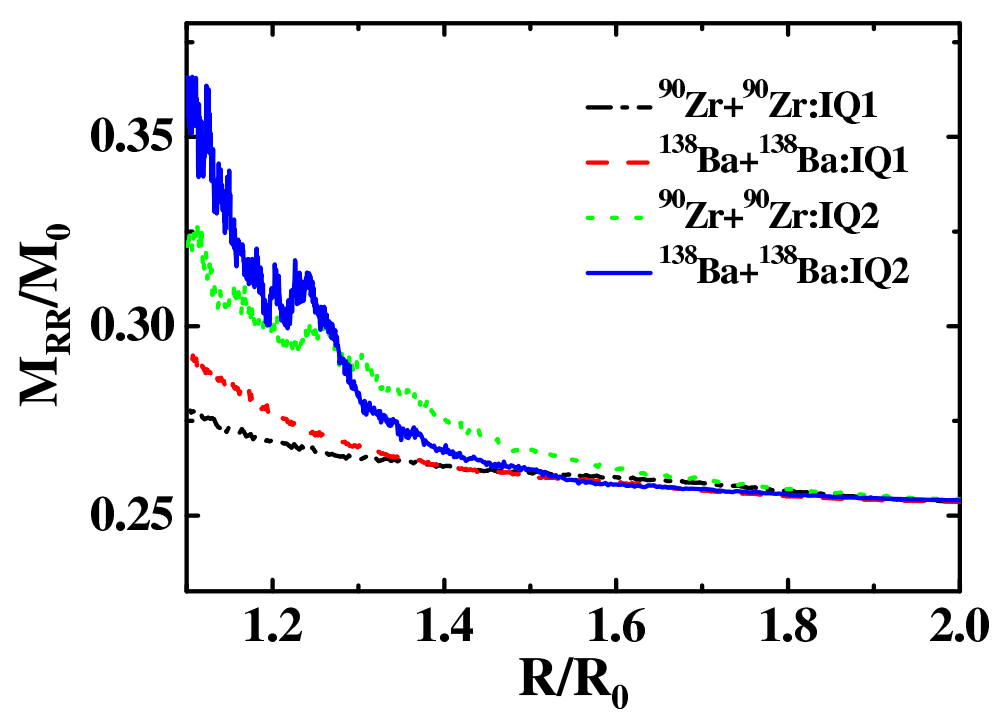

FIG. 4: (Color online)The comparison between mass parameters $M_{R R}$ calculated with force parameter sets IQ1 and IQ2.

for reaction systems of ${ }^{90} \mathrm{Zr}+{ }^{90} \mathrm{Zr}$ and ${ }^{138} \mathrm{Ba}+{ }^{138} \mathrm{Ba}$. One sees that the $M_{R R}$ calculated with IQ1 rises slower than that calculated with IQ2. We notice that the parameter set IQ1 gives relatively smaller incompressibility compared with the force IQ2 (see Table I). The difference between the $M_{R R}$ calculated with IQ1 and IQ2 can be attributed to the difference in incompressibility of interaction parameters IQ1 and IQ2. The softer EoS needs less energy to make two nuclei even closer than touching configuration and thus the mass parameter for relative motion for the fusion reaction system decreases with the decrease of the stiffness of the EoS.

In order to make comparison with other approaches, in Fig.5 we show the results calculated with hydrodynamic model(Werner-Wheeler (W-W) method) $[7,18]$ and with the linear response function theory model(LRFT)[6], in addition to the microscopic transport model approach for the system of ${ }^{110} \mathrm{Pd}+{ }^{110} \mathrm{Pd}$. The results obtained by the ImQMD model with IQ2 and by hydrodynamic model $(\mathrm{W}-\mathrm{W})$ are shown by solid and long dashed curves, respectively. The short dashed curve is the results by LRFT taken from [6] where the large fluctuation appears due to the level crossing. The general tendency of the $M_{R R}$ changing with $\mathrm{R} / \mathrm{R}_{0}$ calculated by the ImQMD model is similar with those obtained by hydrodynamic model and by LRFT. The magnitude of the mass parameter obtained by the ImQMD model is higher than that obtained by hydrodynamic model, but lower than that obtained by LRFT. 


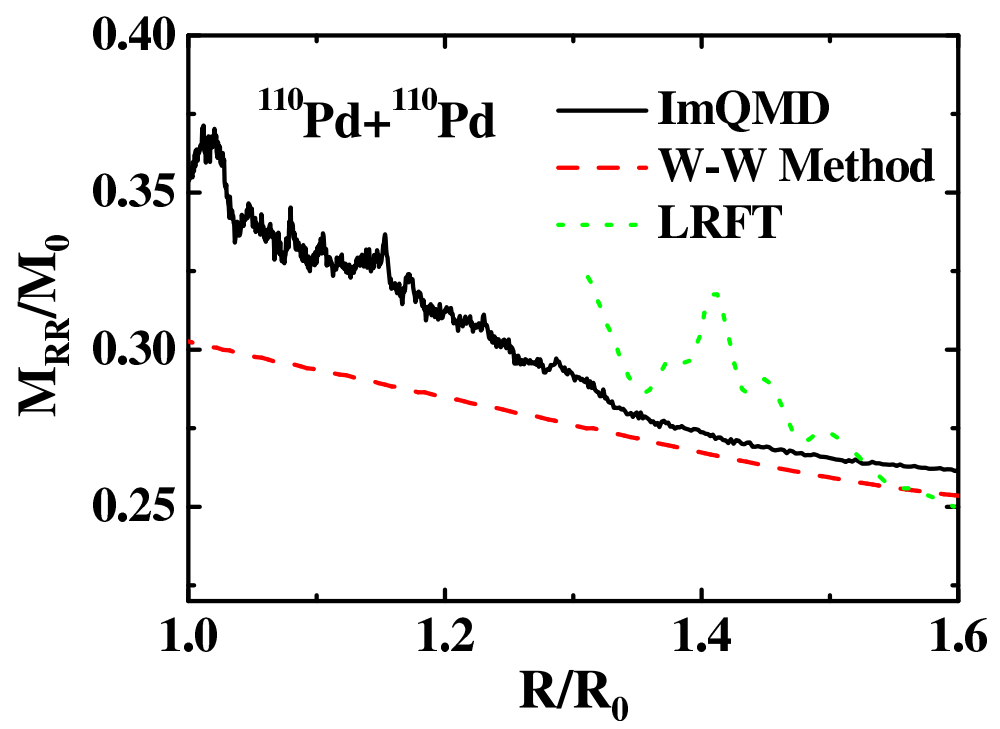

FIG. 5: (Color online)The comparison of mass parameter $M_{R R}$ calculated by the ImMQD model with that by Werner-Wheeler method and the linear response function theory.

\section{B. The mass parameter for the neck motion in fusion reactions}

In order to study the mass parameter for the neck motion within microscopic transport model one has to first define a nuclear surface based on the density distribution of the reaction system. The shape of the nuclear system is then defined by the nuclear surface. The nuclear surface is usually defined by an equi-density surface of the reaction system. In this work this equi-density surface, namely the $\rho_{s}(z, t)$ for a axial symmetric system at time $\mathrm{t}$ is taken to be at the half normal density $\rho=0.5 \rho_{0}$. The neck width $\Delta$ is defined by $2 \rho_{s}\left(z=z_{c}, t\right)$ and the $z_{c}$ denotes the position of the neck as shown in Fig.1. Thus, the distance of two nuclei and neck width are evolved with time simultaneously. Fig.6 shows the correlation between the neck width $\Delta$ and distance between two nuclei $\mathrm{R} / \mathrm{R}_{0}$. The contour plots of density distribution for ${ }^{138} \mathrm{Ba}+{ }^{138} \mathrm{Ba}$ at $\mathrm{R} / \mathrm{R}_{0}=1.9,1.8,1.6$ are also shown in the figure, the lines in the contour plots correspond to $\rho=0.5,0.75,1.0 \rho_{0}$, respectively. One can find that the touching configuration is around $R / R_{0}=1.8$, but not at $R / R_{0}=1.6$ which is about the sum of the radii of two nuclei. This is because two nuclei are elongated due to the interaction between two nuclei. One can further find that the most fast increase of the neck width $\Delta$ happens around the touching configuration, for instance, in the system ${ }^{138} \mathrm{Ba}+{ }^{138} \mathrm{Ba}$ the $\Delta$ from $0.5 \mathrm{fm}$ increases to about $7 \mathrm{fm}$ when the $\mathrm{R} / \mathrm{R}_{0}$ decreases only from 1.9 to 1.6. It means that the touching configuration is important for characterizing the neck 
motion.

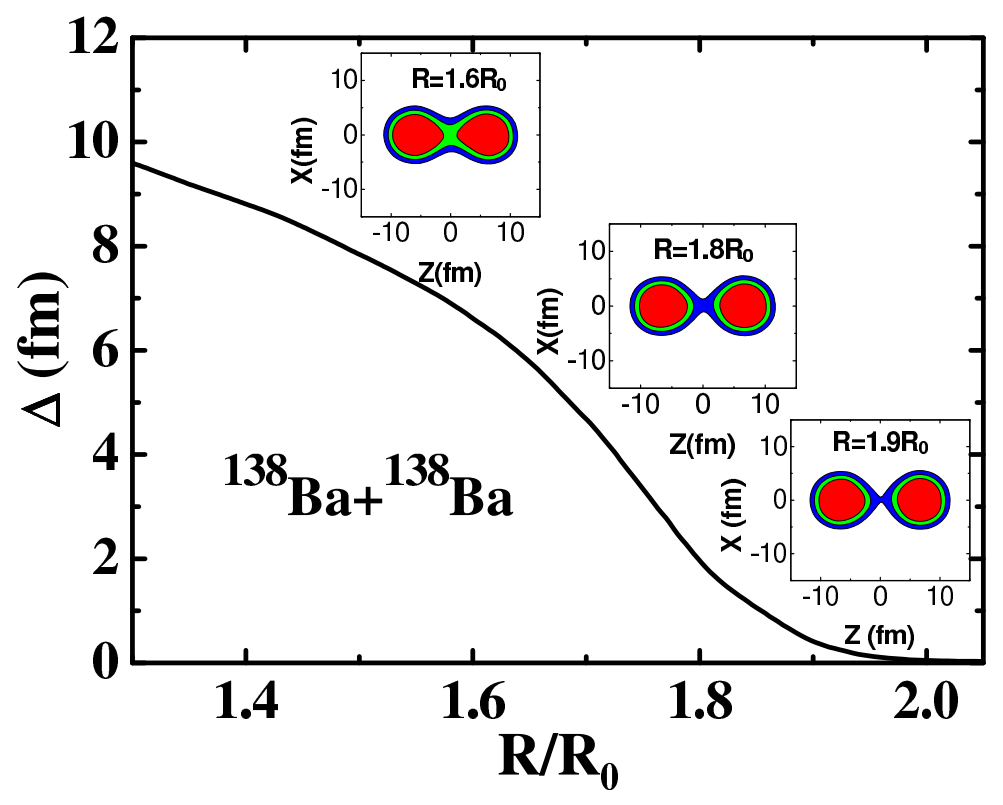

FIG. 6: (Color online)The correlation between neck width $\Delta$ and distance $\mathrm{R} / \mathrm{R}_{0}$ for ${ }^{138} \mathrm{Ba}+{ }^{138} \mathrm{Ba}$.

Mass parameters, in fact, are related with the kinetic energies of collective motions. The collective kinetic energy for relative motion of two colliding nuclei and neck motion can be expressed by

$$
T=T_{R R}+T_{R \Delta}+T_{\Delta \Delta}=\frac{1}{2}\left[M_{R R} \dot{R}^{2}+2 M_{R \Delta} \dot{R} \dot{\Delta}+M_{\Delta \Delta} \dot{\Delta}^{2}\right]
$$

As is indicated in ref.[15] and [5] that for the mass symmetric reactions the mixing mass parameter involving the neck degree of freedom remains comparatively small. It means that the condition $M_{R \Delta} / \sqrt{M_{R R} M_{\Delta \Delta}}<<1$ is approximately satisfied[5]. In order to test whether the crossing term in (20) can be eliminated or not, in Fig.7 we show the time evolution of $\dot{R}$ and $\dot{\Delta}$ for ${ }^{138} \mathrm{Ba}+{ }^{138} \mathrm{Ba}$. It is seen from the figure that the $\dot{\Delta}$ is a strongly peaked function of time, and the peak is at about $350 \mathrm{fm} / \mathrm{c}$ with very steep rising left side, while $\dot{R}$ is a monotonically decreasing function of time. The $\dot{R}$ reduces considerably at $280 \mathrm{fm} / \mathrm{c}$, which is much earlier than the time when the $\dot{\Delta}$ reaches the peak value. From this figure we find that the coupling between the velocities of the relative motion and neck motion is indeed not strong within the time period which we are interested in and thus, neglecting the crossing term in (20) is reasonable. Here we should mention that the velocities for neck and relative motion are strongly correlated when $\mathrm{t}>450 \mathrm{fm} / \mathrm{c}$ seen from the figure. We should also notice that the neck is already well developed at that time and in this case it is difficult to well 
distinguish the relative and neck motion and the treatment of neglecting the crossing term adopted in this work only gives approximate results .

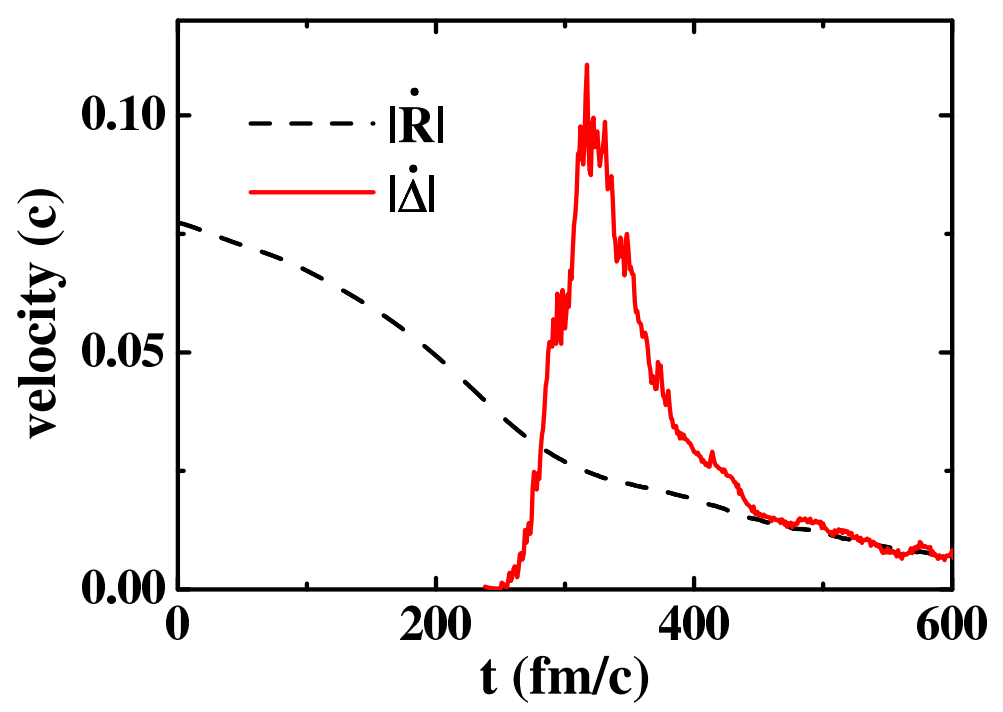

FIG. 7: (Color online)The time evolution of the $\dot{\Delta}$ and $\dot{R}$ for ${ }^{138} \mathrm{Ba}+{ }^{138} \mathrm{Ba}$. The solid curve is for $\dot{\Delta}$ and the dashed curve for $\dot{R}$.

Now let us investigate the mass parameter for the neck motion in symmetric fusion systems. Instead of using the relation of $\mathrm{M}_{\Delta \Delta}=\mathrm{P}_{\Delta} / \dot{\Delta}$ we calculate $\mathrm{M}_{\Delta \Delta}$ through the relation

$$
T_{\Delta \Delta}=\frac{1}{2} M_{\Delta \Delta} \dot{\Delta}^{2}
$$

and

$$
T_{\Delta \Delta}=E_{t o t}-E_{p o t}-T_{R R}-T_{o t h}-E_{e x c} .
$$

Here $T_{\text {oth }}$ is the kinetic energy from the motion of the additional degrees of freedom except the relative motion and neck motion. Since in the early stage of fusion reaction the change of the deformations at two ends of colliding system is not strong, the energy corresponding to this motion could be neglected[6]. Thus the $T_{\text {oth }}$ is mainly from the crossing term of relative motion and neck motion. As is mentioned above this term is small, then the $T_{\text {oth }}$ should be small and could be neglected. The $E_{e x c}$ is the internal excitation energy. As we know that the neck motion plays important role only near touching configuration when the internal excitation is weak, in addition the incident energy is selected to be low, the internal excitation energy should be much smaller than the collective motions. Thus, we neglect the $\mathrm{E}_{\text {exc }}$ in the present calculations for simplicity. The $E_{p o t}$ is calculated by

$$
E_{p o t}(R)=E_{12}(R)-E_{1}-E_{2} .
$$


Here, $E_{12}(R), E_{1}$ and $E_{2}$ are the total energy of the whole system, the energies of the projectile(like) and target (like) part, respectively. The reactions ${ }^{90} \mathrm{Zr}+{ }^{90} \mathrm{Zr}$ and ${ }^{138} \mathrm{Ba}+{ }^{138} \mathrm{Ba}$ at incident energies equal to 1.08 times the height of the Bass barrier $\left(\mathrm{E}_{c m}=350 \mathrm{MeV}\right)$ are selected to study the mass parameter for neck motion. Here the incident energy chosen is lower than that in the investigation of the mass parameter for relative collection motion in order to reduce the internal excitation energy. Fig.8(a) shows the time evolution of kinetic energies for relative and neck motions, and of the potential energy for ${ }^{138} \mathrm{Ba}+{ }^{138} \mathrm{Ba}$ at $\mathrm{E}_{c m}=350 \mathrm{MeV}$. The parameter set IQ2 is utilized. Here the initial time is taken at the time when the surface to surface distance of two nuclei equals to $22.5 \mathrm{fm}$ (correspond to $\mathrm{R} / R_{0}=3.6$ in Fig.8(b)). One sees from the figure that the kinetic energy for relative motion decreases firstly and then approaches to zero with time increasing, while the kinetic energy for neck motion is about zero at beginning, then it increases and until $550 \mathrm{fm} / \mathrm{c}$ it saturates. The potential energy is zero at the infinite distance, then it increases as two nuclei approach with each other and it reaches a maximum value at about $300 \mathrm{fm} / \mathrm{c}$, after then it reduces to a saturated value. In Fig.8(b) we show the kinetic energies for relative motion and neck motion and the potential energy as a function of $\mathrm{R} / \mathrm{R}_{0}$. The energy for relative motion gradually decreases with $\mathrm{R} / \mathrm{R}_{0}$ decreasing and finally equals to zero, while the energy for neck motion starts to increase quickly from zero to a saturated value from $R / R_{0} \sim 1.9$ to $R / R_{0} \sim 1.3$. We find that the magnitude of the kinetic energy for neck motion exceeds that of relative motion just after the touching configuration. The kinetic energies for both relative and neck motion are saturated at about $\mathrm{R} / \mathrm{R}_{0}=1.3$. It is also noticed that the potential energy also reaches a maximum value near the touching configuration.

Having the kinetic energy for neck motion $T_{\Delta \Delta}$ and velocity $\dot{\Delta}$, we can calculate the mass parameter for neck motion through expression (21). Fig.9(a) presents the evolution of the mass parameter for neck motion with the relative distance between the centers of mass of two nuclei and Fig.9(b) shows the mass parameters as a function of the neck width scaled by $\mathrm{L}$, the total length of the colliding system, for the systems ${ }^{90} \mathrm{Zr}+{ }^{90} \mathrm{Zr},{ }^{138} \mathrm{Ba}+{ }^{138} \mathrm{Ba}$, respectively. From Fig.9 one sees that the $M_{\Delta \Delta}$ reaches a small minimum near the touching configuration. Then it increases with decrease of $\mathrm{R} / \mathrm{R}_{0}$ and increase of $\Delta$. We notice the change in the slope of $M_{\Delta \Delta}$ with respect to $\mathrm{R} / \mathrm{R}_{0}$ and $\Delta$ near the touching configuration, which is due to the sharp peak of $\dot{\Delta}$ as shown in Fig.7. The magnitude of the $M_{\Delta \Delta}$ is from less than tenth of the system mass $\left(A_{1}+A_{2}\right)$ to several times the system mass. Finally it 


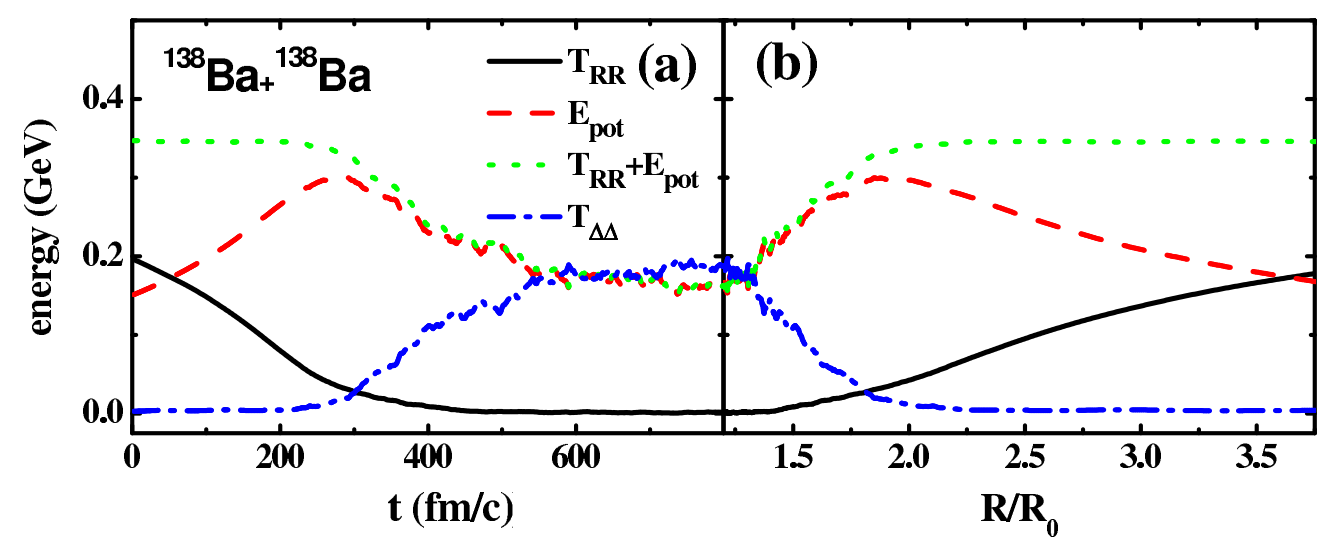

FIG. 8: (Color online)The kinetic energies for the relative and neck motions and the interaction potential energy for the reaction ${ }^{138} \mathrm{Ba}+{ }^{138} \mathrm{Ba}$ at $E_{c m}=350 \mathrm{MeV}$ (a) as a function of time and (b)as a function of $\mathrm{R} / R_{0}$.

approaches to saturate values for both systems of ${ }^{90} \mathrm{Zr}+{ }^{90} \mathrm{Zr}$ and ${ }^{138} \mathrm{Ba}+{ }^{138} \mathrm{Ba}$. The figure shows that slopes for $M_{\Delta \Delta}$ vs $\Delta\left(\mathrm{R} / \mathrm{R}_{0}\right)$ for ${ }^{90} \mathrm{Zr}+{ }^{90} \mathrm{Zr}$ and ${ }^{138} \mathrm{Ba}+{ }^{138} \mathrm{Ba}$ are different and it implies the system dependence of the mass parameter for neck motion. We have also investigated the influence of the effective interaction on the mass parameter for neck motion by comparing the mass parameters calculated with IQ1 and IQ2 and we find that there is no big difference between them. It seems to us that the dependence of the mass parameter for neck motion on the effective interaction is weaker than that for the relative collection motion in nuclear fusion reactions along the $\beta$-stability line.

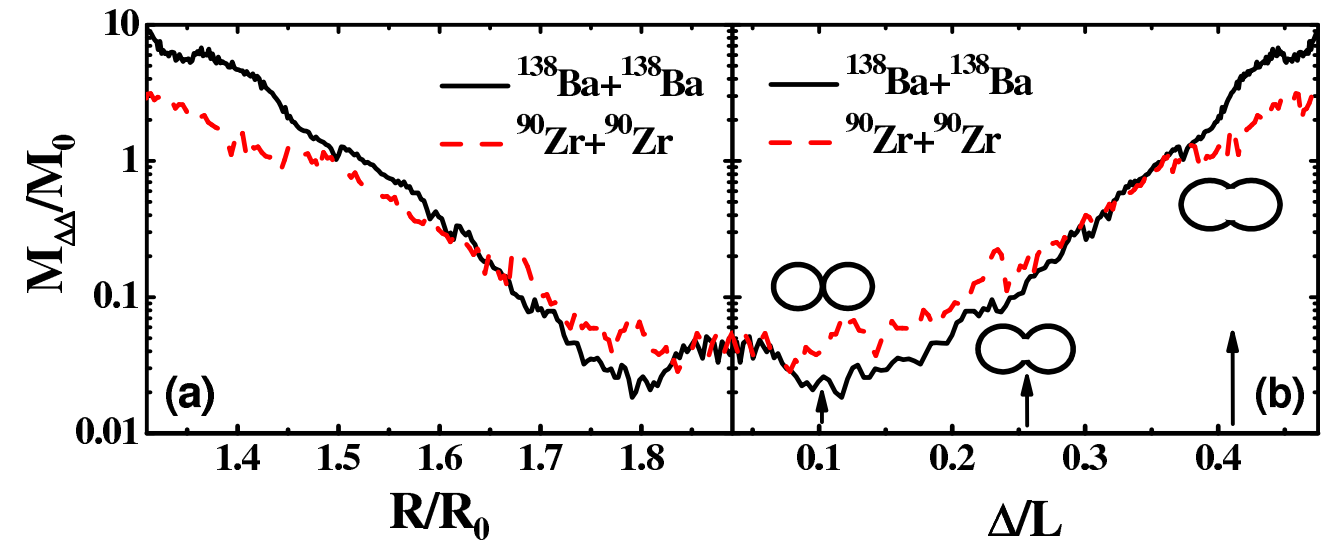

FIG. 9: (Color online)The mass parameter $\mathrm{M}_{\Delta \Delta}$ for the neck motion as a function of a)R/R $\mathrm{R}_{0}$ and b) $\Delta / L$ for systems ${ }^{138} \mathrm{Ba}+{ }^{138} \mathrm{Ba}$ and ${ }^{90} \mathrm{Zr}+{ }^{90} \mathrm{Zr}$ calculated with IQ2.

Finally, we make comparison between the mass parameter for neck motion obtained by means of the microscopic transport model and the hydrodynamic model as well as the 
LRFT. Fig.10 shows $M_{\Delta \Delta} / \mathrm{M}_{0}$ as function of neck widths $\Delta$ at $\mathrm{R} / \mathrm{R}_{0}$ around the touching configuration. Here, the solid, dashed, and dotted lines denote the $M_{\Delta \Delta}$ calculated by the microscopic transport model, the hydrodynamic model and the results taken from ref. [6] calculated by LRFT, respectively. The general behavior of the $M_{\Delta \Delta}$ as a function of neck width is coincident for all results obtained by three different approaches and also is in agreement with other studies[5, 15], i.e. the $M_{\Delta \Delta}$ increases with $\Delta$ and finally it saturates when the neck is already very much developed. The results of this work are in between the results from hydrodynamic model and LRFT approaches. This is similar with the situation in mass parameter for relative motion as shown in Fig.5.

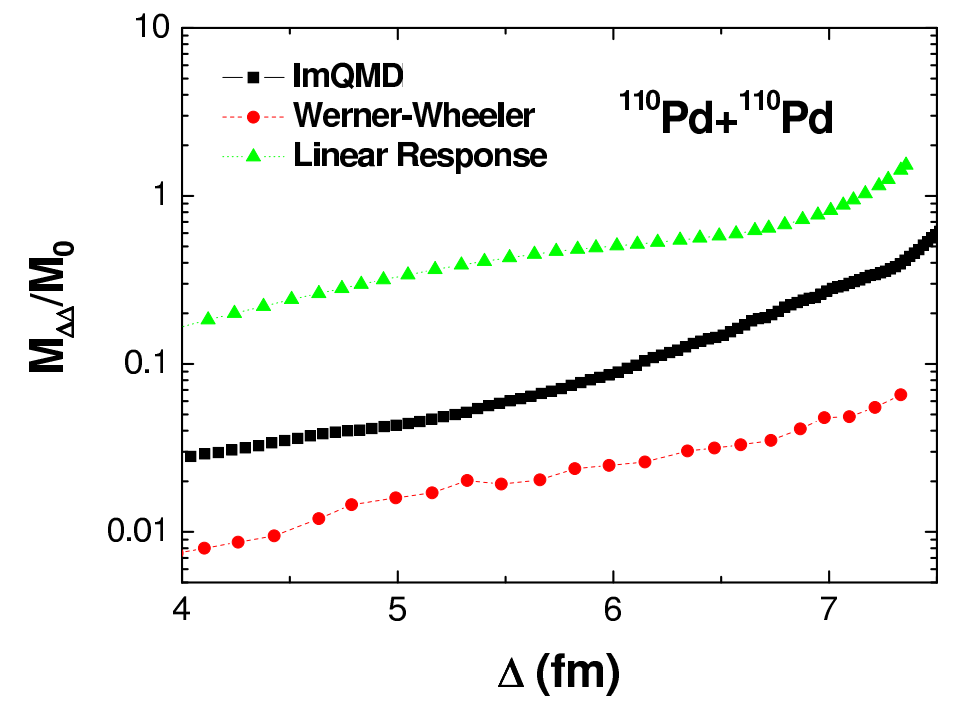

FIG. 10: (Color online)The comparison of the mass parameter $\mathrm{M}_{\Delta \Delta}$ calculated by the ImQMD model with that calculated by hydrodynamic model and by LRFT taken from [6].

\section{SUMMARY AND DISCUSSION}

In summary, in this paper we employ the microscopic transport model, namely the Improved Quantum Molecular Dynamics model to study the mass parameters of collective motions in fusion reactions for the first time. The head on reactions of ${ }^{90} \mathrm{Zr}+{ }^{90} \mathrm{Zr},{ }^{110} \mathrm{Pd}+{ }^{110} \mathrm{Pd}$, and ${ }^{138} \mathrm{Ba}+{ }^{138} \mathrm{Ba}$ are selected. The essential difference of the present approach from other methods is that within a microscopic dynamical model approach, the shape of the reaction system is determined by the time dependent density distribution of the system, so that the 
distance between the centers of mass of two nuclei and the neck width change with time self-consistently during the reaction process. It is found that the feature of the relative

and the neck motion is rather different, and the $\dot{\Delta}$ and the $\dot{R}$ are coupled weakly, as seen from Fig.7. So that we can study the $M_{\Delta \Delta}$ and $M_{R R}$ individually. Then we investigate the mass parameters for the relative and neck motion for three systems. We find that the mass parameter for relative motion between two nuclei approaches the reduced mass when the reaction system is at the separated configuration and after contact of two partners it increases with decrease of the distance between two centers of mass. The mass parameter for neck motion has a small minimum near touching configuration and after touching configuration it increases with the increase of neck width(or the decrease of relative distance between two nuclei). Its magnitude is from less than the tenth to more than several times the total mass of the system. The general tendency of the dependence of $M_{R R}$ on the $\mathrm{R} / R_{0}$ and $M_{\Delta \Delta}$ on $\Delta$ is similar with those obtained by the hydrodynamic model and the LRFT. The magnitude of mass parameters obtained in this work is larger than the ones obtained by the hydrodynamic model and smaller than those obtained by LRFT. Both $M_{R R}$ and $M_{\Delta \Delta}$ depend on the reaction systems. And the influence of the effective interactions on the mass parameters is obvious for the mass parameters $M_{R R}$ but not for $M_{\Delta \Delta}$.

\section{Acknowledgments}

Authors thank Profs. W. Greiner, W. Scheid and Y.Abe for variable discussions. One of authors(Kai Zhao) is grateful to Dr. Caiwan Shen for the help in the calculation of the mass parameter within the hydrodynamic model. This work is supported by the National Natural Science Foundation of China under Grant Nos.10235030,10675172 and National Basic Research Programme of China under contract no. 2007CB209900.

[1] V. M. Strutinsky, Nucl. Phys. A95, 420 (1967).

[2] W. D. Myers, et. al., Nucl. Phys. 81, 1 (1966).

[3] M. D. Brack, et. al., Rev. Mod. Phys.44, 320 (1972).

[4] P. Möller, D. G. Madland, A. J. Sierk, A. Iwamoto, Nature, 409, 785 (2001).

[5] G. G. Adamian, N. V. Antonenko, S. P. Ivanova and W.Scheid, Nucl. Phys. A 646,29 (1999). 
[6] G. G. Adamian, N. V. Antonenko, A.Diaz-Torres and W.Scheid, Nucl. Phys. A 671,233 (2000).

[7] Caiwan Shen, Grigori Kosenko, Yasuhisa Abe,. Phys. Rev. C 66, 061602 (2002).

[8] V. Zagrebaev and W. Greiner, J. Phys. G: Nucl. Phys. 31,825 (2005).

[9] D. R. Inglis, Phys. Rev. 96, 1059 (1954); D. R. Inglis, Phys. Rev. 103, 1786 (1956).

[10] S. T. Belyaev, Mat. Fys. Medd. Dan. Vid. Selsk. 31, 11 (1959).

[11] D. J. Thouless et. al., Nucl. Phys. 21, 225 (1960).

[12] H. Hofmann, Phys. Rep. 284, 139 (1997).

[13] W. Cassing and W. Nörenberg, Nucl. Phys. A 433, 467 (1985).

[14] Renfa Feng, Xizhen Wu, et. al., High energy Phys. and Nucl. Phys. 8, 81 (1984).

[15] V. Schneider, J. Maruhn and W. Greiner, Z. Phys. A 323, 111 (1986).

[16] J. R. Nix, et. al., Ann. Phys. 41, 52 (1967).

[17] F. G. Werner et.al., Unpublished Manuscript (no data), J. R. Nix, et. al., Nucl. Phys. A130, 241 (1969).

[18] Xizhen Wu, Chinese Journal of Nucl. Phys. 9, 145 (1987).

[19] R. A. Gherghescu and D. N. Poenaru, Phys. Rev. C 72, 027602 (2005).

[20] Ning Wang, Zhuxia Li, Xizhen Wu, Phys. Rev. C 65, 064608 (2002).

[21] Ning Wang, Zhuxia Li, Xizhen Wu, and el. al., Phys. Rev. C 69, 034608 (2004).

[22] M. Papa, T. Maruyama, and A. Bonasera,Phys. Rev. C 64, 024612 (2001).

[23] N. Wang, Z.X. Li, X.Z. Wu, et al., Mod. Phys. Lett. A 20, 2619 (2005).

[24] Junlong Tian, Xizhen Wu, Kai Zhao, Yingxun Zhang and Zhuxia Li, Phys. Rev. C 77, 064603 (2008).

[25] Ch.Hartnack, Zhuxia Li, L.Neise, and et.al., Nucl. Phys. A495, 303 (1989); Zhuxia Li, Ch.Hartnack, H.Stoecker and W.Greiner, Phys. Rev. C 40, 824 (1991).

[26] J.Aichelin, Phys. Rep. 202233 (1991), and references therein.

[27] Yingxun Zhang, Zhuxia Li, Phys. Rev. C 74, 014602 (2006).

[28] G. G. Adamian, N. V. Antonenko, R. V. Jolos, Nucl. Phys. A 584, 2059 (1995).

[29] Reiner Bass, Nuclear reactions with heavy ion , New York; Berlin Heiderberg 1980. 